\title{
Agency-Theorie, Informationskosten und Managervergütung
}

\section{Ulrike Graßhoff und Joachim Schwalbach*}

\author{
Humboldt-Universität Berlin zu Berlin, \\ Institut für Management \\ Spandauerstraße 1, 10178 Berlin \\ email:schwal.@wiwi.hu-berlin.de.
}

2. Februar 1999

*Die Arbeit entstand im Rahmen des Teilprojekts C4: "Dynamik des Wettbewerbs" des DFG-Sonderforschungsbereichs 373 der Humboldt-Universität zu Berlin.

Für hilfreiche Anregungen bedanken wir uns bei Brigitte Adolph, Knut Bartels, Anette Boom, Paul Milgrom, Stefan Profit und John Roberts. 


\section{Einleitung}

Die Trennung von Eigentum und Kontrolle ist eines der auffallendsten Merkmale der Unternehmensentwicklung. Daraus kann sich ein Interessenkonflikt zwischen dem Management und den Eigentümern ergeben, wenn das Management bestrebt ist, die Ziele zu verfolgen, die nicht zwangsläufig dem Eigentümerinteresse entsprechen. Die Agency-Theorie leitet einen potentiellen Interessenkonflikt aus den unvollkommen beobachtbaren Präferenzen der Beteiligten ab. Danach kann der Eigentümer das Arbeitsergebnis des Managements beobachten, jedoch nur unvollkommen dessen Anstrengungsniveau und Qualität. Zwar können die Eigentümer Anstrengungen unternehmen, das Verhalten des Managements zu erfassen, es wird dennoch nicht zu einer völligen Transparenz führen, da die Grenzkosten den Grenznutzen übersteigen werden. Als Ausweg bleibt, dem Management ein Anreizsystem anzubieten, das dessen Verhalten im Sinne der Eigentümer steuert. Ein derartiges Anreizsystem ist dann (pareto) optimal, wenn der Eigentümernutzen unter Beachtung des minimalen Reservationsnutzens des Managements maximal ist. Das risikoaverse Management wird dann das Anstrengungsniveau so wählen und seine Fähigkeiten so einsetzen, daß ein im Sinne des Eigentümers zieladäquates Ergebnis erzielt wird.

Zahlreiche empirische Studien haben versucht, die in den Unternehmen vorhandenen monetären Anreizsysteme auf ihre Anreizkompatibilität hin zu untersuchen. Dabei wurden überwiegend lineare Anreizfunktionen unterstellt, wonach sich die Vergütung aus einem festen Bestandteil (Grundgehalt) und einem erfolgsabhängigen Bestandteil (Bonus, Tantieme, Aktienoptionen) zusammensetzt. Obwohl ein optimales Anreizsystem die festen und variablen Bestandteile simultan bestimmt, fand in den empirischen Studien der Anreizintensitätsfaktor der variablen Vergütung besondere Beachtung. Die geschätzten Elastizitäten betrugen für die U.S.A. etwa 0.1-0.15, für Japan etwa 0.01 und für Deutschland etwa $0.06^{1}$. Daraus wurde nahezu übereinstimmend der Schluß gezogen, daß der Intensitätsfaktor zu gering ist, um im Sinne der Agency-Theorie optimal zu sein. Stellvertretend für viele stellten daraufhin Jensen und Murphy ${ }^{2}$ folgendes fest: "...the lack of strong

\footnotetext{
${ }^{1}$ Siehe hierzu die folgenden Studien: Rosen (1992), Kato (1997) und Schwalbach/Graßhoff (1997). Die Ergebnisse variieren entsprechend der verwendeten Maße, Unternehmensgröße und Branche. Dennoch ist die Varianz gering. Vergleiche auch die Unterschiede innerhalb Europas in Conyon/Schwalbach (1997).

${ }^{2}$ Siehe Jensen/Murphy (1990), S. 262.
} 
pay-for-performance incentives for CEOs indicated our evidence is puzzling.".

Obwohl zahlreiche empirische Studien zu nahezu identischen Ergebnissen zur Anreizelastizität kommen, decken sie jedoch eine gewisse Hilflosigkeit mit dem Umgang der als relativ gering angesehenen Werte auf. Der Grund hierfür ist in der fehlenden theoretischen Erklärung niedriger Elastizitäten zu sehen. Rosen ${ }^{3}$ drückt dies wie folgt aus: "...what is not so clear from theory is what a reasonable benchmark would be. Is the 0.1-0.15 elasticity estimate too small or too large? The theory has not focused enough on that number to provide an answer ...". Der vorliegende Beitrag soll diese Lücke schließen und eine theoretische Erklärung für den in den empirischen Studien erzielten niedrigen Anreizintensitätsfaktor geben. Die theoretische Erklärung wird darüber Aufschluß geben, ob die empirischen Ergebnisse in der Tat ein Puzzle darstellen. Weiterhin werden die theoretischen Erkenntnisse für zukünftige empirische Arbeiten nützlich sein.

\section{Theoretische Erklärung zur optimalen Anreizintensität}

Die folgenden Betrachtungen basieren auf dem 'Linear Exponential Normal Model', kurz LEN-Modell, ausführlich beschrieben in Spremann ${ }^{4}$. Dieses Modell formalisiert eine Prinzipal-Agenten-Beziehung, bei der das Unternehmensergebnis $z$ vom Anstrengungsniveau $e$ sowie von Zufallseinflüssen $u$ in der einfachen Form:

$$
z=e+u
$$

abhängt, wobei für die zufällige Größe $u$ eine Normalverteilung mit Erwartungswert $E(u)=0$ und Varianz $\operatorname{Var}(u)=\sigma^{2}<\infty$ angenommen wird. Die Größen $e$ und $u$ sind für den Eigentümer nur unvollkommen beobachtbar. Unter diesen Umständen liegt es im Interesse des Eigentümers, ein Belohnungs- bzw. Vergütungssystem zu entwickeln, in dem die Vergütung $V$ vom erzielten und beobachtbaren Unternehmensergebnis $z$ abhängt. Im LEN-Modell wird eine lineare Vergütung:

$$
V(z)=\alpha+\beta \cdot z
$$

betrachtet, wobei $\alpha$ den fixen Vergütungsanteil und $\beta$ den ergebnisorientierten variablen Vergütungsanteil beschreiben.

\footnotetext{
${ }^{3}$ Siehe Rosen (1992), S. 201.

${ }^{4}$ Siehe Spremann (1987), S. $11 \mathrm{ff}$.
} 
Das Anreizsystem beugt möglichem opportunistischem Verhalten (moral hazard) des Managements vor. Für das Management bietet das Vergütungssystem Anreize, das Anstrengungsniveau $e$ so zu wählen, daß die Vergütung positiv beeinflußt wird.

Im Modell werden der Prinzipal $P$ (Eigentümer) als risikoneutral und der Agent $A$ (Manager) als risikoavers angesehen. Die Nutzenfunktion $G$ des Prinzipalen ist somit linear und sein erwarteter Nutzen berechnet sich in einfacher Form zu:

$$
E(z-V(z))=(1-\beta) e-\alpha
$$

Der Nettonutzen $w$ des Agenten ist die Differenz aus Vergütung und Kostenanteil für das Anstrengungsniveau:

$$
w=\alpha+\beta z-C(e)
$$

wobei $C^{\prime} \geq 0$ und $C^{\prime \prime}>0$ gelten soll. In Zusammenhang mit dem LENModell wird häufig eine quadratische Kostenfunktion der Form:

$$
C(e)=\frac{1}{2} k e^{2} \text { mit } k>0
$$

angenommen. Unter Verwendung einer exponentiellen Nutzenfunktion $U(w)$ mit konstanter absoluter Risikoaversion $r>0$ :

$$
U(w)=-\exp \{-r w\}
$$

wird die Wohlfahrt des Agenten mittels des Sicherheitsäquivalents $S$ :

$$
S=U^{-1}(E(U(w))
$$

formalisiert. Mit den grundlegenden Annahmen von linearer Vergütung, exponentieller Nutzenfunktion und normalverteiltem Umweltzustand berechnet sich $S$ zu:

$$
S=E(V(z))-\frac{1}{2} r \operatorname{Var}(V(z))-C(e)=\alpha+\beta e-\frac{1}{2} r \beta^{2} \sigma^{2}-\frac{1}{2} k e^{2}
$$

Aus der Sicht des Prinzipalen ergibt sich folgendes Optimierungsproblem: 


$$
\max _{\alpha, \beta}[(1-\beta) e-\alpha]
$$

unter den Nebenbedingungen:

$$
\begin{gathered}
\alpha+\beta e-\frac{1}{2} r \beta^{2} \sigma^{2}-\frac{1}{2} k e^{2} \geq U_{0} \\
e=\arg \max _{\widetilde{e}}\left[\alpha+\beta \widetilde{e}-\frac{1}{2} r \beta^{2} \sigma^{2}-\frac{1}{2} k \widetilde{e}^{2}\right]
\end{gathered}
$$

Die Bedingung (4) besagt, daß dem Agenten ein Mindestnutzen $U_{0}$ sicher ist. Da eine Verringerung des fixen Anteils $\alpha$ eine Erhöhung des Nutzens des Prinzipalen bewirkt, ist diese Ungleichung in dem Optimierungsprogramm mit Gleichheit erfüllt. Die Anreizkompatibilitätsbedingung (5) berücksichtigt die Nutzenmaximierung des Agenten und soll sicherstellen, daß bei gegebener 'moral hazard'-Situation der Agent den Anreiz hat, das gewünschte Anstrengungsniveau $e^{*} \mathrm{zu}$ wählen.

Im folgenden betrachten wir First-best- und Second-best-Lösungen für das LEN-Modell im Vergleich ${ }^{5}$. Dabei setzen wir ohne Beschränkung der Allgemeinheit $U_{0}=0$.

Der First-best-Fall (Optimierung nur mit Bedingung (4)) liefert eine konstante Vergütungsstruktur $V_{A}^{F}$ für den Agenten. Für $e=e^{*}$ folgt:

$$
V_{A}^{F}(z)=\frac{1}{2} k e^{* 2}, \quad e^{*}=\frac{1}{k} .
$$

Der erwartete Nutzen (Ertrag) für den Prinzipalen, $\Pi_{P}^{F}$, ist dann:

$$
\Pi_{p}^{F}=e^{*}-\frac{1}{2} k e^{* 2}=\frac{1}{2 k}
$$

Für den Second-best-Fall (Optimierung mit (4) und (5)) folgt wegen (5) die Bedingung:

$$
\beta=C^{\prime}(e)=k e
$$

\footnotetext{
${ }^{5}$ Vergleiche Holmström/Milgrom (1987).
} 
und mit dieser Beziehung erhält man im Optimum schließlich den Zusammenhang:

$$
\beta^{*}=\left[1+C^{\prime \prime}\left(e^{*}\right) r \operatorname{Var}(u)\right]^{-1}=\left[1+k r \sigma^{2}\right]^{-1} .
$$

Da jetzt $e^{S *}=\frac{\beta^{*}}{k}$ gilt, erhält man mit (4):

$$
\alpha^{*}=\frac{\beta^{*}\left(1-2 \beta^{*}\right)}{2 k} .
$$

Eingesetzt in (3 ) folgt für den Ertrag im Second-best-Fall:

$$
\Pi_{P}^{S}=\frac{\beta^{*}}{2 k}
$$

Die Werte des optimalen variablen Vergütungsanteils $\beta^{*}\left(0<\beta^{*}<1\right)$ und des optimalen Ertrags für den Prinzipalen $\Pi_{P}^{S}$ sind durch den Ausdruck $k r \sigma^{2}$ bestimmt und sind jeweils monoton fallend mit steigenden Werten $k r \sigma^{2}$. Das Verhältnis von $e^{F *} \mathrm{zu} e^{S *}$ bzw. das von $\Pi_{P}^{F}$ zu $\Pi_{P}^{S}$ ist jeweils $\beta^{*}$. Für den Fall, daß die Optimierung eine Lösung $\beta^{*} \approx 1$ ergibt, erhält man eine Annäherung an die First-best-Lösung, was aus einem geringen Wert für das Produkt $k r \sigma^{2}$ resultieren muß. Berücksichtigt man das bekannte Ergebnis, daß das Prinzipal-Agenten Problem bei risikoneutralem Agenten eine dem First-best-Fall äquivalente Lösung besitzt, dann ist es intuitiv klar, daß bei einem genügend kleinem Grad an Risikoaversion eine Lösung nahe der First-best-Lösung ermittelt wird ${ }^{6}$. Außerdem ist es plausibel, daß bei einem hinreichend kleinen Wert für den die Umweltsituation repräsentierenden $\mathrm{Pa}$ rameter $\sigma^{2}$ eine Lösung nahe der des First-best-Falls erzielt wird ${ }^{7}$.

Um die Möglichkeiten der Annäherung der Second-best-Lösung an die First-best-Lösung darzustellen, betrachten wir ein Prinzipal-Agenten-Modell mit linearer Vergütungsstruktur unter Berücksichtigung einer Informationsvariable von Milgrom und Roberts ${ }^{8}$. Für das hier betrachtete Modell wird die Vergütungsstruktur in (2) wie folgt erweitert:

$$
V(z, y)=\alpha+\beta(z+\gamma y)
$$

\footnotetext{
${ }^{6}$ Siehe Milgrom/Roberts (1992).

${ }^{7}$ Entsprechendes gilt für die Opportunitätskosten.

${ }^{8}$ Siehe Milgrom/Roberts (1992), S. $215 \mathrm{ff}$.
} 
wobei

$$
z=e+u
$$

ist. Dabei besitzen $(u, y)$ eine gemeinsame Normalverteilung mit $E(y)=0$, $\operatorname{Var}(y)=\delta^{2}$ und den Korrelationskoeffizienten $\rho$. Für die Kovarianz zwischen den stochastischen Größen $u$ und $y$ im Modell folgt somit:

$$
\operatorname{Cov}(u, y)=\sigma \delta \rho
$$

Die Informationsvariable $y$ liefert für die Vertragsgestaltung zusätzliche Informationen, die beispielsweise aus vergleichbaren Situationen gewonnen werden können. Der Parameter $\gamma$ gewichtet den Einfluß der Informationsvariablen auf die Vergütung und steuert dadurch den Einfluß der Umweltsituation auf die Vergütung. Da das Unternehmensergebnis $z$ von der jeweiligen Umweltsituation beeinflußt wird, kann ein hohes Anstrengungsniveau durch die Umweltsituation überkompensiert werden.

Löst man das zugehörige Agency-Problem ${ }^{9}$, so folgt:

$$
\begin{aligned}
& \beta^{*}=\left[1+k r \sigma^{2}\left(1-\rho^{2}\right)\right]^{-1} \\
& \gamma^{*}=-\frac{\operatorname{Cov}(u, y)}{\operatorname{Var}(y)}=-\frac{\sigma \rho}{\delta}
\end{aligned}
$$

und für das Anstrengungsniveau $e^{*}$ sowie für den im Optimum erzielten erwarteten Ertrag:

$$
e^{*}=\frac{\beta^{*}}{k} \quad \text { und } \quad \Pi_{P}^{S}=\frac{\beta^{*}}{2 k}
$$

In diesem erweiterten Modell ist der Fall $\beta^{*}=1$ enthalten, allerdings nur dann, wenn $\rho^{2}=1$ gilt, bzw. wenn $y$ und $u$ perfekt korreliert sind. In diesem Fall wird die First-best-Lösung erreicht.

Das Modell zeigt, daß durch die Berücksichtigung eines zusätzlichen Signals $y$ eine Varianzreduktion von $\sigma^{2}$ auf $\sigma^{2}\left(1-\rho^{2}\right)$ erzielt werden kann. Die reduzierte Varianz entspricht der bedingten Varianz von $u$ gegeben $y$, die wegen (1) gleich der bedingten Varianz von $z$ gegeben $y$ ist. Sie führt bei

\footnotetext{
${ }^{9}$ Siehe Milgrom/Roberts (1992), S. 219-222.
} 
$\rho^{2}>0 \mathrm{zu}$ einer Erhöhung des variablen Vergütungsanteils und letztlich zu einer Steigerung des Ertrags des Prinzipals.

Die Lösung für $\gamma$ ist $\gamma^{*}=-\frac{\sigma \rho}{\delta}$. Der Gewichtungsparameter $\gamma=0$ kann somit nur dann optimal sein, wenn zwischen der Umweltsituation und der Informationsvariablen keine Korrelation besteht. In dieser Situation ist die Informationsvariable für den Anreizvertrag wertlos. In allen anderen Fällen trägt der Gewichtungsparameter der Beeinflussung durch die Informationsvariablen Rechnung.

Fassen wir die Ergebnisse an dieser Stelle zusammen: Im einfachen Agency-Modell ist der optimale Anreizintensitätsfaktor $\beta^{*}=1$, wenn die Informationsvariable $y$ die Umweltunsicherheit vollkommen erfaßt $\left(\rho^{2}=1\right)$. Je unvollkommener die Unsicherheit erfaßbar ist, desto geringer wird $\beta$ sein. Sehr kleine Werte von $\beta$, wie sie in den empirischen Studien ermittelt wurden, können die Ursache hoher und unvollkommen erfaßbarer Umweltunsicherheiten, ausgeprägter Risikoaversion und/oder hoher Opportunitätskosten des Managers sein. Der Zusammenhang der Einflußfaktoren auf den Anreizintensitätsfaktor wird in Abbildung 1 graphisch verdeutlich. Demnach fällt der Anreizintensitätsfaktor deutlich bei zunehmender Umweltunsicherheit und Risikoaversion.

\section{Das Agency-Modell mit Informationskosten}

Bei der bisherigen Betrachtung wurde davon ausgegangen, daß keine Kosten für die Informationsbeschaffung über die Umweltunsicherheit für den Eigentümer entstehen. Geht man von dem wirklichkeitsnäheren Fall aus, daß Informationen zum Anstrengungsniveau und zur Umweltsituation nicht kostenlos zu erhalten sind, so stellt sich die Frage, in welchen Fällen sich die Informationsgewinnung für den Eigentümer rentiert.

Die Informationsvariable $y$ ist für den Eigentümer von größerem Nutzen je höher der absolute Wert der Korrelation zwischen $u$ und $y$ ist. Es ist plausibel, daß die Kosten für die Information von der Korrelation zwischen $u$ und $y$ abhängen und symmetrisch um $\rho=0$ sind. Im folgenden werden die Betrachtungen von oben erweitert, indem eine auf dem Intervall $[0,1]$ definierte Kostenfunktion $K$ in Abhängigkeit der Größe $\rho^{2}$ im Modell berücksichtigt wird, wobei $x:=\rho^{2}$ gelten soll. Die Kostenfunktion $K$ soll folgende Eigenschaften haben: 
Abbildung 1: Zusammenhang zwischen $\beta^{*}$ und $\rho^{2}$
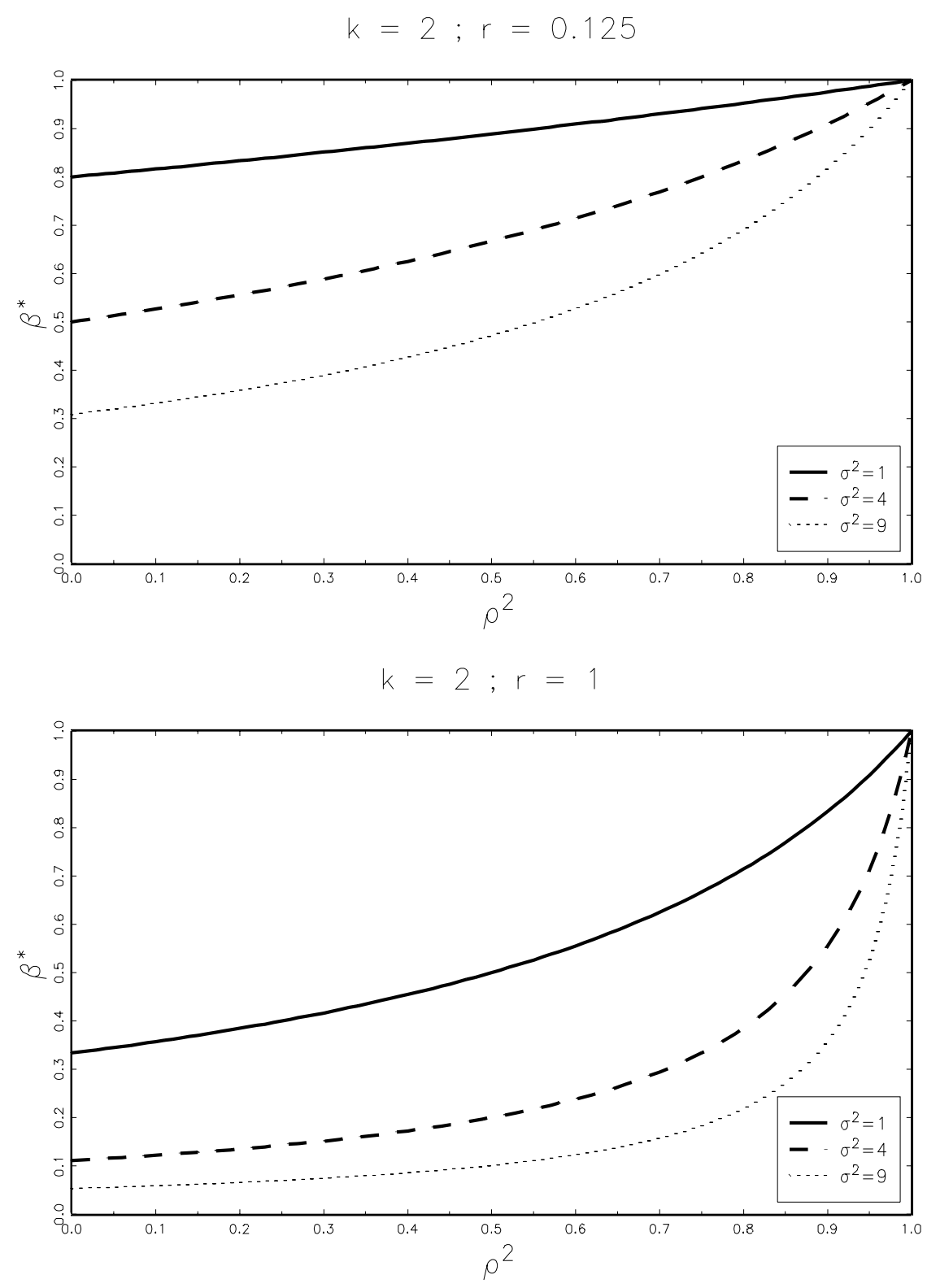


$$
\begin{array}{rlrl}
K(0) & =0 & K(x) \geq 0, & 0<x \leq 1 \\
K^{\prime}(0) \geq 0 & K^{\prime}(x)>0, & 0<x \leq 1 \\
K^{\prime \prime}(x)>0, & 0<x \leq 1 &
\end{array}
$$

Die relevante Ausgangssituation sei wie folgt beschrieben:

Es sei $s:=k r \sigma^{2}$ für gegebene Werte von $k, r$ und $\sigma^{2}$, und wir betrachten den Spezialfall $k=2$.

Dies führt auf der Basis der obigen Ergebnisse ohne Informationsvariable $y$ zu einem optimalen variablen Anteil:

$$
\beta_{0}^{*}(s):=(1+s)^{-1}
$$

und mit der Informationsvariable $y$ jedoch ohne Informationskosten zu:

$$
\beta_{M}^{*}(x ; s):=(1+s(1-x))^{-1}
$$

wobei mit dieser Notation $^{10}$ :

$$
\begin{aligned}
& \beta_{M}^{*}(x ; s)>\beta_{0}^{*}(s) \quad \text { für } 0<x<1 \\
& \beta_{M}^{*}(0 ; s)=\beta_{0}^{*}(s) \\
& \beta_{M}^{*}(1 ; s)=1
\end{aligned}
$$

gilt. Die entsprechenden Erträge des Prinzipalen sind jetzt:

$$
\Pi_{M}(x ; s):=\frac{1}{4} \beta_{M}^{*}(x ; s)-K(x)
$$

Wir setzen für $s>0$ :

$$
\Pi_{0}(s):=\Pi_{M}(0 ; s)=\frac{1}{4} \beta_{0}^{*}(s) .
$$

Für gegebenen Wert $s>0$ hängt der Verlauf der Funktion $\Pi_{M}(\cdot ; s)$ von der zugrunde gelegten Kostenfunktion ab. Unter den gegebenen Voraussetzungen kann $\Pi_{M}(\cdot ; s)$ streng monoton wachsend oder fallend sein, jedoch auch lokale

\footnotetext{
${ }^{10}$ Der Index 'M' steht für den Fall: 'Mit Informationsvariable'.
} 
Maxima und/oder lokale Minima annehmen.

Existieren keine lokalen Maxima, so folgt sofort $x_{s}^{O P T}=0$ oder $x_{s}^{O P T}=1$ je nachdem, ob:

$$
K(1) \gtrless \frac{1}{4}\left(\beta_{M}^{*}(1 ; s)-\beta_{0}^{*}(s)\right)=\frac{s}{4(1+s)}
$$

gilt.

Die Bedingungen 1. und 2. Ordnung für die Maximierung von (7) sind:

$$
\frac{s}{4(1+s(1-x))^{2}}=K^{\prime}(x)
$$

und

$$
\frac{s^{2}}{2(1+s(1-x))^{3}}<K^{\prime \prime}(x),
$$

Ist die Kostenfunktion der Gestalt, daß bei gegebenem $s>0$ Lösungen $x_{s}^{*}$ von ( 8 ) und (9) für $0<x<1$ existieren, so lauten die Bedingungen für den Vergleich mit den Funktionswerten der Randpunkte:

$$
\Pi_{M}\left(x_{s}^{*} ; s\right)>\Pi_{0}(s)=\frac{1}{4(s+1)}
$$

und

$$
\Pi_{M}\left(x_{s}^{*} ; s\right)>\Pi_{M}(1 ; s)=\frac{1}{4}-K(1)
$$

Sind diese erfüllt, so folgt: $x_{s}^{O P T}=x_{s}^{*}$.

Schreibt man Ungleichung (10) in der Form:

$$
\frac{1}{4\left(1+s\left(1-x_{s}^{*}\right)\right.}-\frac{1}{4(s+1)}>K\left(x_{s}^{*}\right)
$$

und setzt man für die Differenz auf der linken Seite die Bedingung 1.Ordnung (8) für $x_{s}^{*}$ ein, so läßt sich die Ungleichung (10) zu:

$$
K^{\prime}\left(x_{s}^{*}\right)\left(1-\frac{s x_{s}^{*}}{1+s}\right)>\frac{K\left(x_{s}^{*}\right)-K(0)}{x_{s}^{*}}
$$


umwandeln. Mit entsprechenden Umformungen läßt sich Ungleichung (11) in:

$$
K^{\prime}\left(x_{s}^{*}\right)\left(1+s\left(1-x_{s}^{*}\right)\right)<\frac{K(1)-K\left(x_{s}^{*}\right)}{1-x_{s}^{*}} .
$$

überführen.

Hinreichende Bedingungen dafür, daß die Randlösungen $x_{s}^{O P T}=0 \mathrm{bzw}$. $x_{s}^{O P T}=1$ ausgeschlossen werden können, berechnen sich in diesem Optimierungsproblem zu:

$$
\begin{gathered}
K^{\prime}(0)<\frac{s}{4(1+s)^{2}} \Longrightarrow x_{s}^{O P T}>0 \\
K^{\prime}(1)>\frac{s}{4} \Longrightarrow x_{s}^{O P T}<1
\end{gathered}
$$

Eine vollständige Charakterisierung der globalen Maxima hängt von der jeweils zugrunde gelegten Kostenfunktion ab. Anhand exemplarischer Betrachtungen werden wir im folgenden verschiedene Möglichkeiten darstellen. Im Vordergrund steht die Ausgangsfrage: In welchen Fällen rentiert sich die Informationsgewinnung? Mit anderen Worten: Es werden die Bedingungen für $x_{s}^{O P T}=0$ gesucht.

Zunächst behandeln wir kurz ein Beispiel zur linearen Kostenfunktion ${ }^{11}$, was sich dadurch auszeichnet, daß keine lokalen Maxima existieren. Mit dem zweiten Beispiel zeigen wir, daß $x_{s}^{O P T}>0$ für alle $s>0$ vorliegen kann. Das dritte Beispiel ist so konstruiert, daß die Kosten für höheren Informationsgehalt unbeschränkt anwachsen und im Grenzfall der vollständigen Information nicht mehr definiert sind.

\subsection{Beispiel 1: $K(x)=a x, a>0$}

Unter Verwendung einer linearen Kostenfunktion der Form $K(x)=a x$, mit $a>0$ zeigt sich, daß die Bedingungen 1. und 2. Ordnung für $s>0$ keine Lösungen im Intervall ]0,1[ besitzen, da die Bedingung 2. Ordnung für $0<x<1$ nie erfüllt ist. Ein Vergleich von $\Pi_{M}(x, s):=\frac{1}{4} \beta_{M}^{*}(x ; s)-K(x)$ für $x=0$ und $x=1$ liefert bei $s>0$ :

\footnotetext{
${ }^{11}$ Diese Kostenfunktion erfüllt die Ausgangsbedingung $K^{\prime \prime}(x)>0$ nicht. Um den Sachverhalt der Randlösungen deutlich zu machen, haben wir den Fall der linearen Kostenfunktion mit aufgenommen.
} 


$$
\begin{array}{lll}
a>\frac{s}{4(1+s)} & \quad & x_{s}^{O P T}=0 \\
a<\frac{s}{4(1+s)} \quad & \Longrightarrow & x_{s}^{O P T}=1
\end{array}
$$

Betrachtet man dies unter dem Aspekt, daß ein gegebener Wert $s>0$ einem variablen Vergütungsanteil $\beta_{0}^{*}=\frac{1}{1+s}$ ohne Berücksichtigung von $y$ entspricht, so zeigt sich, daß wenn der Kostenfaktor $a>\frac{1-\beta_{0}^{*}}{4}$, d.h. wenn $a$ größer als die Differenz der Erträge unter First- und Second-best- Lösung ohne Informationskosten ist, so bringt die Verwendung von $y$ keine Ertragssteigerung für den Prinzipal.

\subsection{Beispiel 2: $K(x)=b x^{2}, b>0$}

Für die Kostenfunktion $K(x)=b x^{2}$ mit $b>0$ gilt im Gegensatz zum vorherigen Beispiel: $K^{\prime}(0)=0$. Damit gilt nach (14) sofort $x_{s, b}^{O P T}>0$. Das heißt, mit einer Informationsvariablen $y$, die den Informationsgehalt $\rho^{2}=x_{s, b}^{O P T}$ hat, kann der Prinzipal den maximalen Ertrag bei Informationskosten erzielen und die Informationsgewinnung lohnt sich.

Im Anhang unter 5.1 sind in Abbildung 5 Verläufe der Ertragsfunktion $\Pi_{M}(\cdot ; s, b)$ unter quadratischer Kostenfunktion am Beispiel $s=\frac{1}{4}$ und $s=1$ für verschiedene Werte von $b>0$ graphisch dargestellt. Weiterhin findet man dort eine Abgrenzung der Fälle, bei denen die Verwendung einer Informationsvariablen, die vollständige Information enthält, optimal ist.

\subsection{Beispiel 3: $K(x)=c \frac{x}{1-x}, c>0$}

Dieses Beispiel unterscheidet sich von den bisherigen Fällen dadurch, daß die Kostenfunktion $K(x)$ nur für $0 \leq x<1$ definiert, und auf diesem Intervall nach oben unbeschränkt ist. Damit ist $x_{s}^{O P T}<1$, d.h. eine Informationsvariable mit vollständiger Information bringt für kein $s>0$ Vorteile. Weiterhin besitzt diese Kostenfunktion positive Grenzkosten im Nullpunkt, so daß $x_{s}^{O P T}=0$ nicht auszuschließen ist. Im folgenden werden wir uns auf eine Zusammenfassung der Ergebnisse zu diesem Beispiel beschränken. Eine ausführlichere Herleitung findet man im Anhang unter 5.2.

Aus den Bedingungen 1. und 2. Ordnung folgt bei gegebenen Werten $s>0$ und $c>0$ für $x_{s, c}^{O P T}$ : 


$$
\begin{aligned}
& c<\frac{s}{4(1+s)^{2}} \Longrightarrow x_{s, c}^{O P T}=x_{s, c}^{*} \\
& c \geq \frac{s}{4(1+s)^{2}} \Longrightarrow x_{s, c}^{O P T}=0
\end{aligned}
$$

mit

$$
x_{s, c}^{*}=1-\frac{2 \sqrt{c s}}{s(1-2 \sqrt{c s})} .
$$

Für gegebenen Kostenfaktor $c>0$ läßt sich $x_{s, c}^{O P T}$ als Funktion in $s>0$ als:

$$
x_{c}^{O P T}(s)= \begin{cases}x_{s, c}^{*} & \underline{s}_{c}<s<\bar{s}_{c} \\ 0 & \text { sonst }\end{cases}
$$

schreiben, wobei sich die Intervallgrenzen $\underline{s}_{c}$ und $\bar{s}_{c}$ aus der Bedingung $c<$ $\frac{s}{4(1+s)^{2}}$ ableiten. In Abbildung 2 ist der Verlauf von $x_{c}^{O P T}(\cdot)$ für verschiedene Werte $c>0$ graphisch dargestellt. Die Abbildungen 3 und 4 zeigen die entsprechenden Funktionen $\beta_{c}^{O P T}(\cdot)$ und $\Pi_{c}^{O P T}(\cdot) .^{12}$

In dem Intervall $\left[\underline{s}_{c}, \bar{s}_{c}\right]$ sind die optimalen Werte $x_{c}^{O P T}(s)$ positiv, die optimalen Anreizintensitäten und Erträge größer als die ohne Verwendung der Informationsvariable. Für Werte von $s>0$ außerhalb des Intervalls ist $x_{c}^{O P T}(s)=0$, und die Funktionswerte $\beta_{c}^{O P T}(s)$ und $\Pi_{c}^{O P T}(s)$ sind mit $\beta_{0}^{*}(s)$ bzw. $\Pi_{0}^{*}(s)$ identisch. Somit rentiert sich die Verwendung der Informationsvariable in diesen Bereichen nicht.

Betrachten wir dazu die Veränderung in dem Kostenfaktor $c$, so folgt, da die Intervallängen $\bar{s}_{c}-\underline{s}_{c}$ monoton fallend in $c$ sind: Je geringer der Kostenfaktor $c$, um so größer das Intervall für $s$, in dem die Lösung $x_{s, c}^{*}$ nach (16) existiert, bzw. sich eine Informationsgewinnung lohnen kann.

Zum Abschluß formulieren wir die Ergebnisse wie im ersten Beispiel in Abhängigkeit von $\beta_{0}^{*}$, dem variablen Vergütungsanteil, der sich ohne Verwendung von $y$ berechnet. Aus der Bedingung $c<\frac{s}{4(1+s)^{2}}$ folgt mit $s=\frac{1-\beta_{0}^{*}}{\beta_{0}^{*}}$ jetzt die Bedingung:

$$
c<\frac{1}{4} \beta_{0}^{*}\left(1-\beta_{0}^{*}\right)
$$

\footnotetext{
${ }^{12}$ Die Funktionen $\beta_{c}^{O P T}(\cdot)$ und $\Pi_{c}^{O P T}(\cdot)$ sind im Anhang unter 5.2 berechnet.
} 
Für kleine vorliegende Werte $\beta_{0}^{*}$ und für Werte $\beta_{0}^{*}$, die nahe bei Eins sind, steht jeweils nur ein Bereich mit geringen Werten $c$ zur Verfügung, bei denen eine von Null verschiedene optimale Korrelation, $x_{c}^{O P T}\left(\frac{1-\beta_{0}}{\beta_{0}}\right)$, existiert, die mittels $y \mathrm{zu}$ einer Ertragssteigerung führt. Im Fall eines vorgegebenen Wertes $\beta_{0}^{*}=\frac{1}{2}$ folgt allerdings, daß der Ertrag bei Berücksichtigung einer Informationsvariablen $y$ mit einer Korrelation von $\rho^{2}=\frac{1-4 \sqrt{c}}{1-2 \sqrt{c}}$ immer maximal erhöht werden kann. $\left(\beta_{0}=\frac{1}{2}\right.$ resultiert aus der Kombination $\left.\frac{1}{r}=\sigma^{2}\right)$

\section{Schlußfolgerung}

Die Ausführungen haben gezeigt, daß die Agency-Theorie in der Lage ist, die in zahlreichen empirischen Studien ermittelten geringen Werte des Anreizintensitätsfaktors zu erklären. Im Standardmodell sind es Umweltunsicherheiten, Risikoaversion und Opportunitätskosten, die für eine Erklärung herangezogen werden können. Wird zusätzlich noch eine Informationsvariable im Modell berücksichtigt, kann dies zu höheren Anreizintensitätswerten führen und gleichzeitig den Nutzen für den Eigentümer erhöhen. Das optimale Anreizsystem hängt entscheidend von der unterstellten Kostenfunktion für die Informationsbeschaffung ab.

Wenn nun unsere theoretischen Ergebnisse zeigen, daß die empirischen Ergebnisse kein Puzzle im Sinne von Jensen und Murphy ${ }^{13}$ darstellen, welche Implikationen haben sie dann für die empirische Forschung? Zunächst müßten die empirischen Modelle stärker theoriegeleitet sein und Variablen berücksichtigen, die Umweltunsicherheiten, Risikoaversion und Opportunitätskosten abbilden können. Darüber hinaus müßte die Informationsvariable mit der jeweiligen Kostenstruktur in die Modelle aufgenommen werden. Informationskosten werden häufig auch Kontrollkosten genannt. Zwar können diese Kosten nur unvollkommen erfaßt werden, aber ihre Berücksichtigung kann sowohl für die Erklärung des Anreizintensitätsfaktors als auch zur Entwicklung von optimalen Anreizsystemen verwendet werden. Derartige Modelle mit Informationskosten können zeigen, ob die Informationsbeschaffung, beispielsweise über die Kontrollorgane der Unternehmen, im Interesse der Eigentümer ist. Dadurch könnte auch ein Beitrag zur öffentlichen Diskussion über die Effizienz der Unternehmenskontrolle geleistet werden.

\footnotetext{
${ }^{13}$ Siehe Jensen/Murphy (1990).
} 


\section{Anhang}

5.1 Ergänzungen zu Beispiel 2: $K(x)=b x^{2}, b>0$

Für die hier betrachtete quadratische Kostenfunktion ist die Gleichung (8) (Bedingung 1. Ordnung) eine kubische Gleichung, und die Anzahl der reellen Lösungen (Vorzeichen der Diskriminante) ist durch die Bedingung:

$$
b \lesseqgtr \frac{27 s^{2}}{32(1+s)^{3}}
$$

bestimmt. Die Berechnung von lokalen Maxima $x_{s, b}^{*}$ für $s, b>0$ eingeschränkt auf das Intervall ]0, 1[ unter Berücksichtigung der Ungleichung (9) macht eine Fallunterscheidung für $s \leq \frac{1}{2}$ und $s>\frac{1}{2}$ notwendig. Der Vergleich mit den Randlösungen mittels (10) und (11) führt insgesamt zu folgendem Ergebnis: 
Abbildung 2: Optimale Korrelationen $x_{C}^{O P T}(s)$

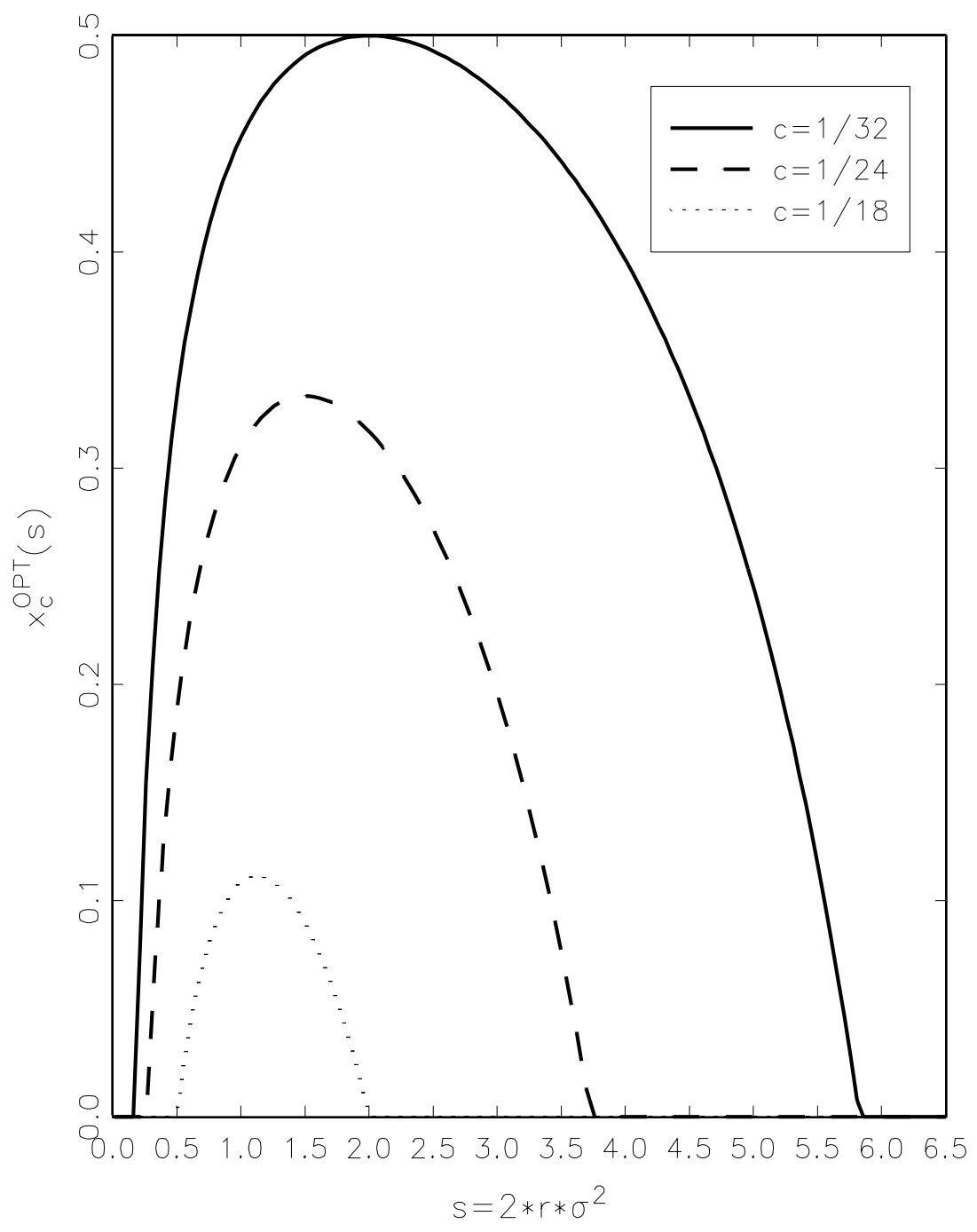


Abbildung 3: Optimale Anreizintensitäten $\beta_{C}^{O P T}(s)$

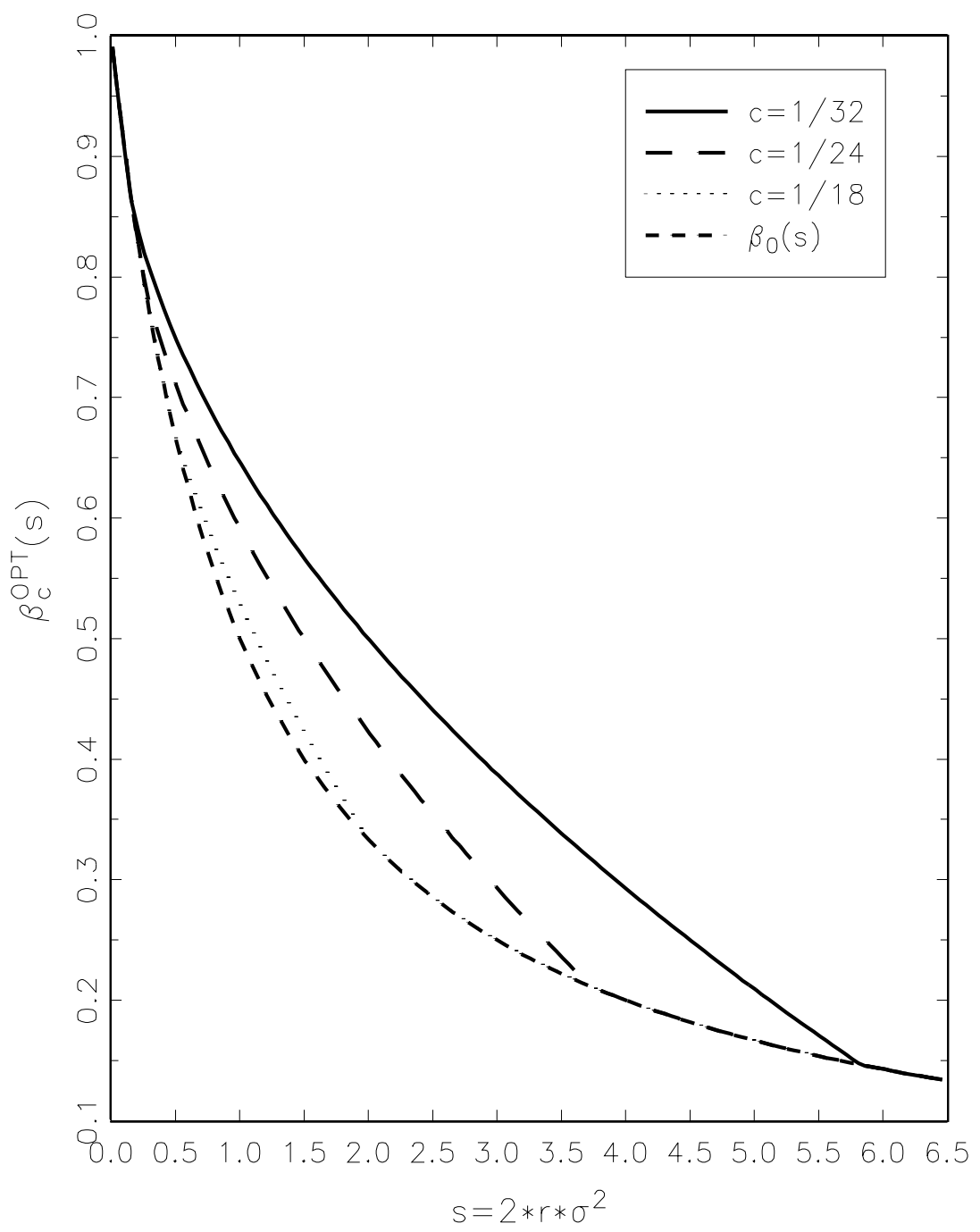


Abbildung 4: Optimale Ertragswerte $\pi_{C}^{O P T}(s)$

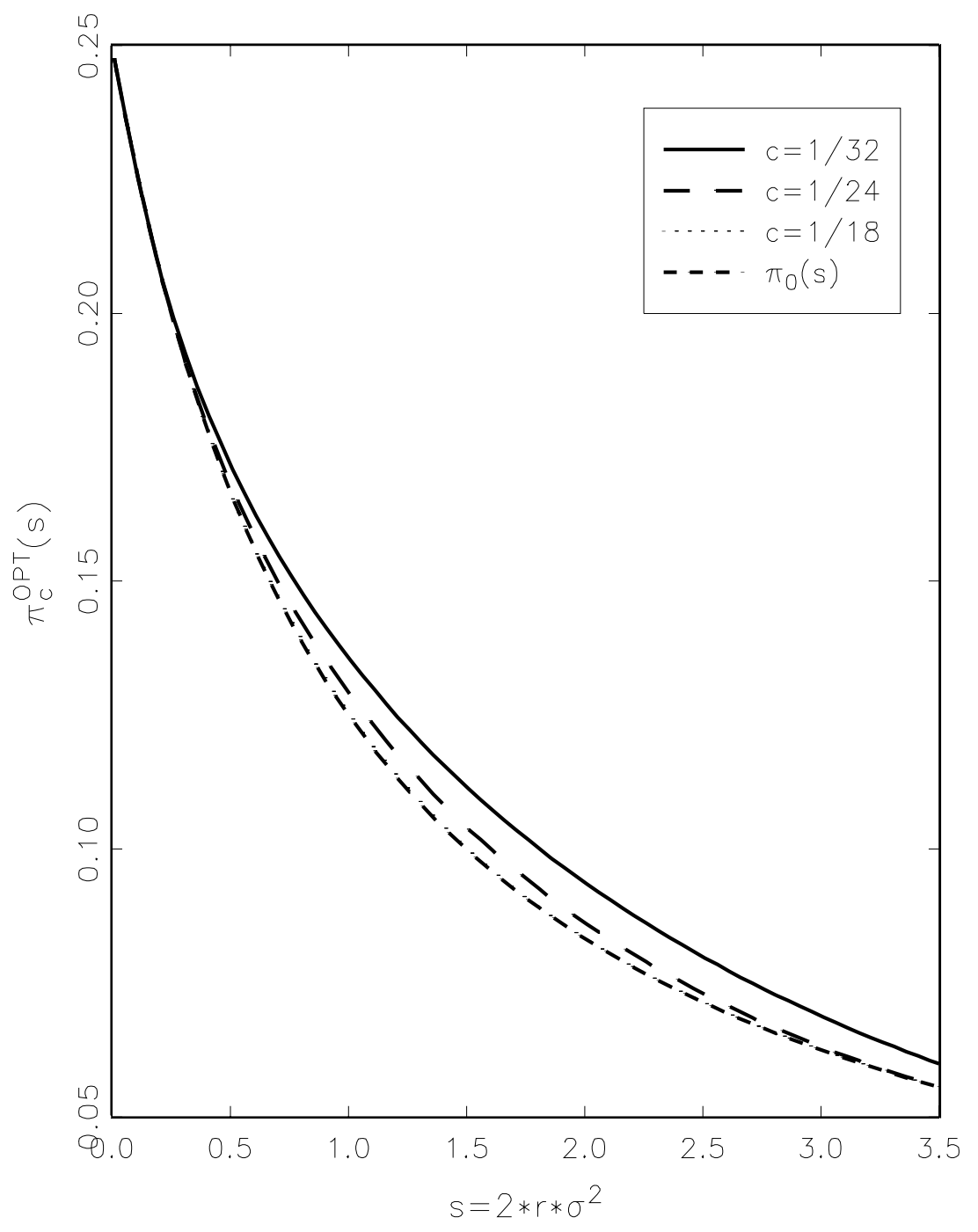


Tabelle 1: Lösungen $x_{s, b}^{O P T}$ bei quadratischer Kostenfunktion

\begin{tabular}{|c|c|c|}
\hline$s \leq \frac{1}{2}$ & $s>\frac{1}{2}$ & $x_{s, b}^{O P T}$ \\
\hline \hline$b \leq \frac{s}{8}$ & $b<\frac{s^{2}}{(1+2 s)^{2}}$ & $x_{s, b}^{O P T}=1$ \\
\hline$b>\frac{s}{8}$ & $b \geq \frac{s^{2}}{(1+2 s)^{2}}$ & $x_{s, b}^{O P T}=x_{s, b}^{*}$ \\
\hline- & $b=\frac{s^{2}}{(1+2 s)^{2}}$ & $x_{s, b}^{O P T}=1 \neq x_{s, b}^{O P T}=\frac{1}{2 s}$ \\
\hline
\end{tabular}

\subsection{Ergänzungen zu Beispiel 3: $K(x)=c \frac{x}{1-x}, c>0$}

In diesem Beispiel führt die Ableitung der Funktion:

$$
\Pi_{M}(x ; s)=\frac{1}{4} \beta_{M}^{*}(x ; s)-K(x)=\frac{1}{4(1+s(1-x))}-\frac{c x}{1-x}
$$

nach der Variable $x$ für gegebenes $s$ und $c$ zu folgender Bedingung 1. Ordnung:

$$
\frac{s}{4(1+s(1-x))^{2}}-\frac{c}{(1-x)^{2}}=0
$$

und weiterhin zur Bedingung 2. Ordnung:

$$
\frac{s^{2}}{2(1+s(1-x))^{3}}-\frac{2 c}{(1-x)^{3}}<0
$$

Gleichung (17) besitzt nur dann eine Lösung $x_{s, c}^{*}$ im Intervall ]0,1[, wenn $c<\frac{s}{4(1+s)^{2}}$. Diese ist eindeutig und lautet:

$$
x_{s, c}^{*}=1-\frac{2 \sqrt{c s}}{s(1-2 \sqrt{c s)}}
$$


Abbildung 5: Ertragsfunktion $\Pi_{M}(. ; s, b)$

$s=0.25$

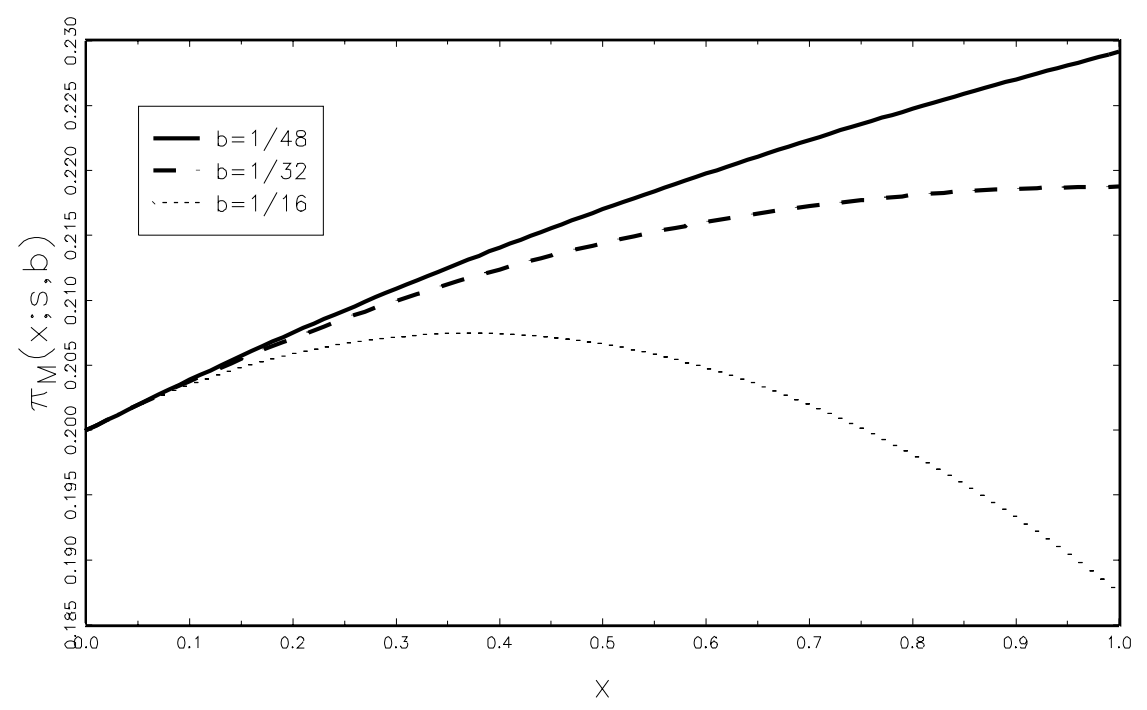

$s=1$

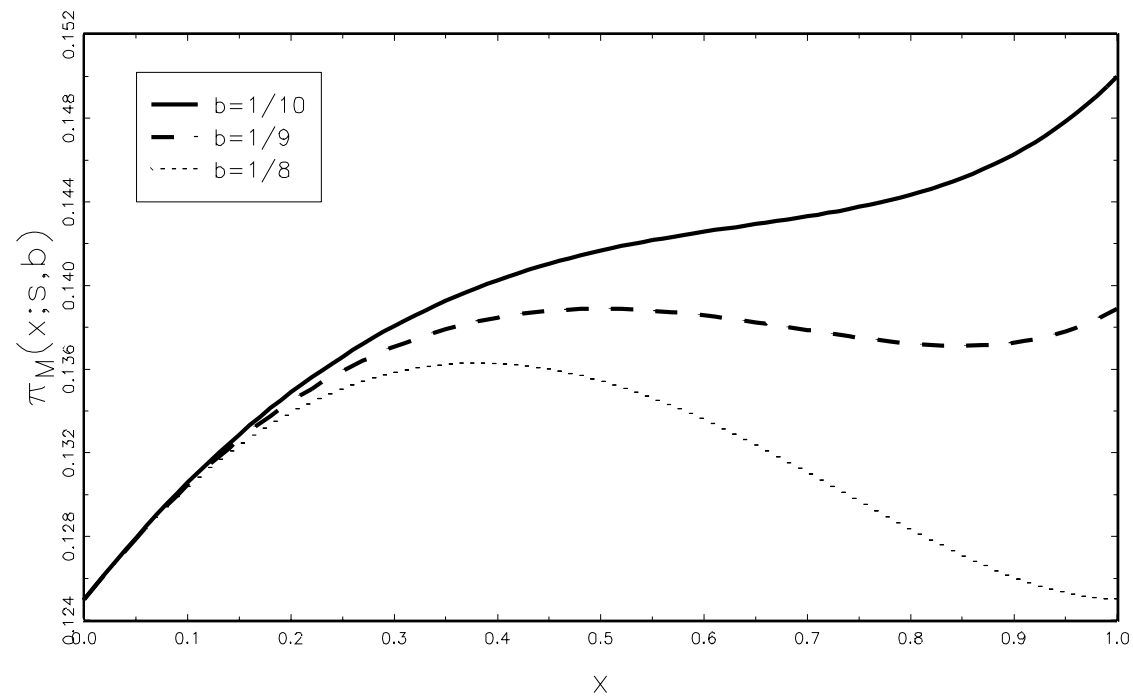


Die Bedingung 2. Ordnung (18) ist für $x_{s, c}^{*}$ erfüllt.

Zusammenfassend folgt:

$$
\begin{aligned}
& c<\frac{s}{4(1+s)^{2}} \Longrightarrow x_{s, c}^{O P T}=x_{s, c}^{*} \\
& c \geq \frac{s}{4(1+s)^{2}} \Longrightarrow x_{s, c}^{O P T}=0
\end{aligned}
$$

Die Bedingung $c<\frac{s}{4(1+s)^{2}}<\frac{1}{16}$ führt zu:

$$
\underline{s}_{c}<s<\bar{s}_{c}
$$

mit:

$$
\underline{s}_{c}=\frac{1-8 c}{8 c}-\frac{\sqrt{1-16 c}}{8 c} \text { und } \bar{s}_{c}=\frac{1-8 c}{8 c}+\frac{\sqrt{1-16 c}}{8 c},
$$

das heißt, für $s$ Werte außerhalb dieses Bereiches folgt dann die Randlösung $x_{s, c}^{O P T}=0$.

Als Funktion in $s>0$ für gegebenes $c>0$ wird $x_{s, c}^{O P T}$ somit zu:

$$
x_{c}^{O P T}(s)= \begin{cases}x_{s, c}^{*} & \underline{s}_{c}<s<\bar{s}_{c} \\ 0 & \text { sonst }\end{cases}
$$

Die Funktion $x_{c}^{O P T}(\cdot)$ besitzt ein lokales Maximum bei $s^{*}=\frac{1}{16 c}$.

Berechnet man mit (19) entsprechend den optimalen variablen Vergütungsanteil und den optimalen Ertrag für gegebenes $c>0$ als Funktionen in $s>0$, so folgt:

$$
\beta_{c}^{O P T}(s)=\left\{\begin{array}{cc}
1-2 \sqrt{s c} & \underline{s}_{c}<s<\bar{s}_{c} \\
\beta_{0}^{*}(s) & \text { sonst }
\end{array}\right.
$$

und

$$
\Pi_{c}^{O P T}(s)=\left\{\begin{array}{cc}
\frac{1}{4}-\sqrt{s c}+s c+c & \underline{s}_{c}<s<\bar{s}_{c} \\
\Pi_{0}^{*}(s) & \text { sonst }
\end{array}\right.
$$

Die Funktionen $\beta_{c}^{O P T}(\cdot)$ und $\Pi_{c}^{O P T}(\cdot)$ sind jeweils monoton fallend in $s$. Für die Intervallänge $l(c)=\bar{s}_{c}-\underline{s}_{c}$ betrachtet als Funktion in $0<c<\frac{1}{16}$ folgt: 


$$
l(c)=\frac{\sqrt{1-16 c}}{4 c}
$$

Da $l^{\prime}(c)=\frac{8 c-1}{4 c^{2} \sqrt{1-16 c}}<0$ für $0<c<\frac{1}{16}$ gilt, ist $l$ eine monoton fallende Funktion.

Betrachten wir die Ergebnisse in Abhängigkeit von $\beta_{0}^{*}$, so läßt sich zunächst aussagen, daß bei $c>\frac{1}{16}$ die Verwendung von $y$ keine Ertragssteigerung bringt. Ist $c<\frac{1}{16}$, so wird für $\beta_{0}^{*}$ im Bereich:

$$
\frac{1}{2}-\sqrt{\frac{1}{4}-4 c}<\beta_{0}^{*}<\frac{1}{2}+\sqrt{\frac{1}{4}-4 c}
$$

mit $x_{s, c}^{*}$ eine Ertragssteigerung erzielt, wobei $s=\frac{1-\beta_{0}^{*}}{\beta_{0}^{*}} \mathrm{zu}$ setzen ist. Das angegebene Intervall ist äquivalent zu der Bedingung:

$$
c<\frac{1}{4} \beta_{0}^{*}\left(1-\beta_{0}^{*}\right)
$$

\section{Literatur}

Conyon, Martin and Joachim Schwalbach (1997), European Differences in Executive Pay and Corporate Governance, Forschungsbericht Nr.97-7, Institut für Management, Humboldt-Universität zu Berlin.

Holmström, Bengt and Paul Milgrom (1987), Aggregation and Linearity in the Provision of Intertemporal Incentives, Econometrica, Vol. 55, March, S. 303-328.

Jensen, Michael C. and Kevin J. Murphy (1990), Performance Pay and Top-Management Incentives, Journal of Political Economy, Vol. 98, No. 2, April, S. 225-264.

Kato, Takeo (1997), Chief Executive Compensation and Corporate Groups in Japan: New Evidence from Micro Data, in: Harry Barkema, Paul A. Geroski und Joachim Schwalbach (eds.), Managerial Compensation, Strategy and Firm Performance, International Journal of Industrial Organization, Vol. 15, No. 4, July, special issue, S. 455-467. 
Milgrom, Paul and John Roberts (1992), Economics, Organization and Management, Englewood Cliffs: Prentice Hall.

Rosen, Sherwin (1992), Contracts and the Market for Executives, in: Lars Wernin and Hans Wijkander, eds., Contract Economics, Oxford: Basil Blackwell, S. 181-211.

Schwalbach, Joachim und Ulrike Graßhoff (1997), Managervergütung und Unternehmenserfolg, Zeitschrift für Betriebswirtschaft, 67. Jg., Nr. 2, Februar, S. 203-217.

Spremann, Klaus (1987), Agent and Principal, in: Günter Bamberg und Klaus Spremann (Hrsg.), Agency Theory, Information, and Incentives, Berlin. Springer, S. 3-37. 


\section{Summary}

Agency theory assumes a potential conflict of interest between principal and agent. Principals provide incentives to agents to influence agents' behaviour in the interest of the principal. In practice, incentive systems are dominanted by financial incentives and consist of fixed and performance related variable pay. Empirical studies concentrated on the analysis of the sensitivity of variable pay. Most studies found very low values which were considered too low to have any incentive effect.

This paper tries to give a theoretical answer to the empirical puzzle of low incentive factors. In the first step, we show that low values of the incentive factor occur if high environmental uncertainty, high risk aversion by the agent and/or high opportunity costs for the agent exist. In the second step, we show that investments in information gathering by the principal to reduce uncertainty lead to higher incentive factors as well as higher returns for the principal. The magnitude of improved returns is shown by assuming various information cost functions. 